\title{
Directed percolation phase transition to sustained turbulence in Couette flow
}

\author{
Grégoire Lemoult $^{1 \dagger}$, Liang Shi ${ }^{1,2 \dagger}$, Kerstin Avila ${ }^{1,2}$, Shreyas V. Jalikop ${ }^{1}$, Marc Avila ${ }^{3}$ and Björn Hof ${ }^{1 \star}$
}

Turbulence is one of the most frequently encountered nonequilibrium phenomena in nature, yet characterizing the transition that gives rise to turbulence in basic shear flows has remained an elusive task. Although, in recent studies, critical points marking the onset of sustained turbulence ${ }^{1-3}$ have been determined for several such flows, the physical nature of the transition could not be fully explained. In extensive experimental and computational studies we show for the example of Couette flow that the onset of turbulence is a second-order phase transition and falls into the directed percolation universality class. Consequently, the complex laminar-turbulent patterns distinctive for the onset of turbulence in shear flows ${ }^{4,5}$ result from short-range interactions of turbulent domains and are characterized by universal critical exponents. More generally, our study demonstrates that even high-dimensional systems far from equilibrium such as turbulence exhibit universality at onset and that here the collective dynamics obeys simple rules.

In basic shear flows (pipe, channel, Couette and boundary layer flows) the onset of turbulence is characterized by the sudden occurrence of chaotic domains ${ }^{4}$ that coexist and compete with laminar motion, resulting in irregular continually changing flow patterns (Fig. 1). In this first manifestation, turbulence is sustained, yet intrinsically patchy, and the state of space-filling fully turbulent motion typical for larger Reynolds numbers is not yet stable ${ }^{6}$. The earliest attempts to explain the initial appearance of turbulence were largely concerned with the linear stability of the laminar flow ${ }^{7-10}$, and later studies took the potential of perturbations to exhibit transient growth ${ }^{11,12}$ into account. More recently, much progress has been made regarding the microscopic flow structures (building blocks) underlying the chaotic motion ${ }^{13-18}$; however, these advances have provided limited insights into the macroscopic transition process. Analogous to phase transitions in thermodynamics it may be expected that the nature of the transition is instead determined by large-scale correlations which do not depend on the details of the microscopic interactions but rather on their collective behaviour. Such concepts are well established for phase transitions in equilibrium statistical mechanics; turbulence, on the other hand, is dissipative and hence not in equilibrium. Although, generally, non-equilibrium systems are much more abundant, there is surprisingly little experimental confirmation of predicted universal characteristics ${ }^{19-21}$.

Possible analogies between the onset of turbulence and certain classes of non-equilibrium phase transitions, more specifically the so-called directed percolation (DP) universality class, were first pointed out 30 years ago by Pomeau ${ }^{22}$. A simple realization of DP is bond percolation. Here, an active state can spread by means of connected bonds on a fixed grid with a probability $P$, and proliferation can take place only by nearest-neighbour interaction. Similarly, the spreading of turbulence in shear flows is a contact process: owing to the linear stability of the laminar state, turbulence cannot arise spontaneously, but only through interaction with domains that are already turbulent. Percolation is governed solely by the probability $P$ and, analogously, the transition to sustained turbulence, and the likelihood of turbulence to spread, is governed by a single parameter-in this case the Reynolds number, $\mathrm{Re}=U h / v$, where $U$ is half the wall speed difference, $h$ half the gap between the walls and $v$ the kinematic viscosity. In DP there is an exact critical point, $P=P_{c}$, below which the system will always return to the passive state (Fig. 1a, left panel), whereas at $P=P_{c}$ (Fig. 1a, mid panel) a continuous phase transition occurs and for $P>P_{c}$ (Fig. 1a, right panel) the fraction of active states is strictly greater than zero (in the thermodynamic limit). There are certain requirements that need to be fulfilled for DP, such as the absence of long-range interactions, the absence of additional symmetries or conservation laws, and the existence of a unique absorbing state (the laminar state in Couette flow qualifies as an absorbing state owing to its linear stability). Indeed, it has been conjectured that these are not only necessary but also sufficient criteria for DP (refs 23,24). More generally, the DP universality class is believed to play a central role for non-equilibrium phenomena, ranging from transport in porous media to the spreading of epidemics or forest fires ${ }^{19}$. However, so far only a single experimental verification for the rather specific case of liquid crystal electro-convection ${ }^{25}$ has been possible.

Regarding a possible connection between turbulence and DP, analogies have been discussed in a variety of studies considering model systems $\mathrm{s}^{26-30}$. However, in the only detailed experimental study, a discontinuous first-order transition was reported, disagreeing with the continuous DP type transition scenario. These experiments were carried out for Couette flow, where a fluid is sheared between two moving walls ${ }^{31,32}$. Equally, simulations of the same flow ${ }^{33,34}$ reported a non-universal first-order transition.

More recently, however, in an investigation of pipe flow ${ }^{1}$ the critical point for the onset of sustained turbulence could be determined and it was shown that the time and length scales relevant close to the critical point are much longer than expectedand, in particular, also much larger than those considered in refs 31-34. In the case of pipe flow these excessively long timescales make it virtually impossible to determine the nature of the transition. An analogous study was performed for Couette flow ${ }^{2}$ in a slender (spanwise) but long (streamwise) domain, and here the relevant timescales (that is, characteristic decay and spreading times of turbulent stripes) close to the critical point are three orders of magnitude smaller than in pipes. Resolving these scales

${ }^{1}$ IST Austria, Am Campus 1, 3400 Klosterneuburg, Austria. ${ }^{2}$ Max Planck Institute for Dynamics and Self-Organization, Bunsenstrasse 10, 37073 Göttingen, Germany. ${ }^{3}$ Friedrich-Alexander-Universität Erlangen-Nürnberg, 91058 Erlangen, Germany. ${ }^{\dagger}$ These authors contributed equally to this work.

*e-mail: bhof@ist.ac.at 
a

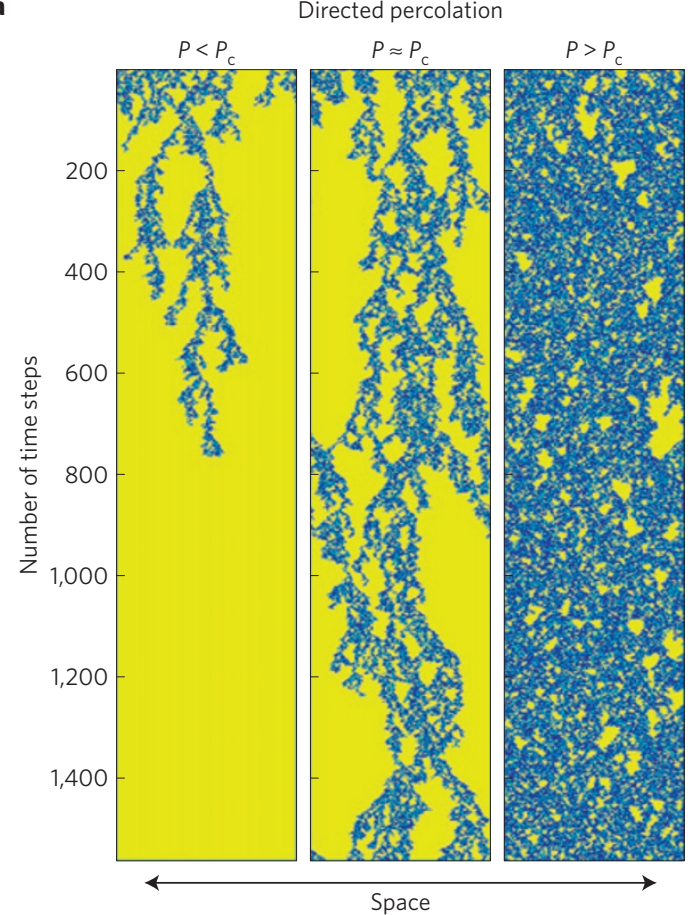

b

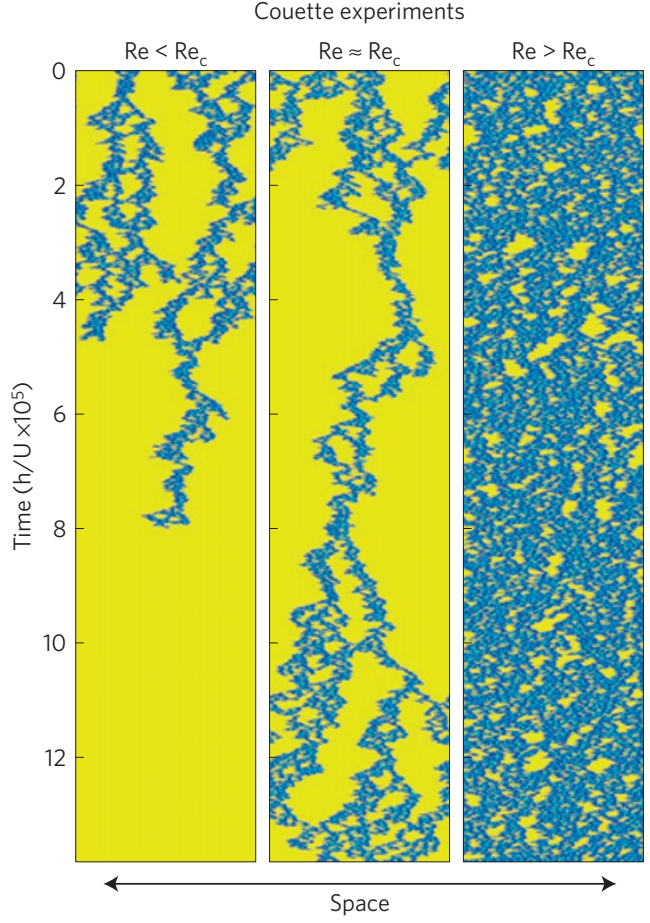

Figure 1 | Spatio-temporal dynamics in experiments of turbulent Couette flow and in DP. a, Simulations of bond directed percolation below, at and above the critical probability, $P_{c}$. Here, active sites are shown in blue and the absorbing/laminar state is shown in yellow. Below the critical point all sites eventually enter the absorbing state (left panel). Close to the critical point (mid panel), active sites persist, yet large laminar regions appear (scale invariance). Finally, for $P>P_{c}$ (right panel), active sites fill a large fraction of the domain. $\mathbf{b}$, Space-time plots are shown for Couette flow below, at and above the critical point. Horizontal lines correspond to snapshots of the flow field in the experiment at a given time (laminar regions are shown in yellow and turbulent regions are shown in blue). Below the critical point (left panel) turbulence disappears after sufficiently long times. At the critical point (mid panel) turbulence survives, but only occupies a small fraction of the domain. Sufficiently far above the critical Reynolds number (right panel) turbulent patches occupy most of the flow domain.

a

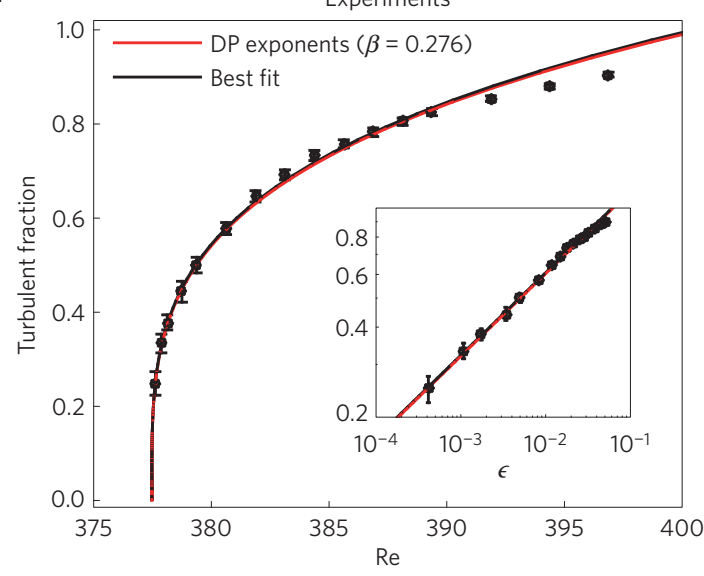

b

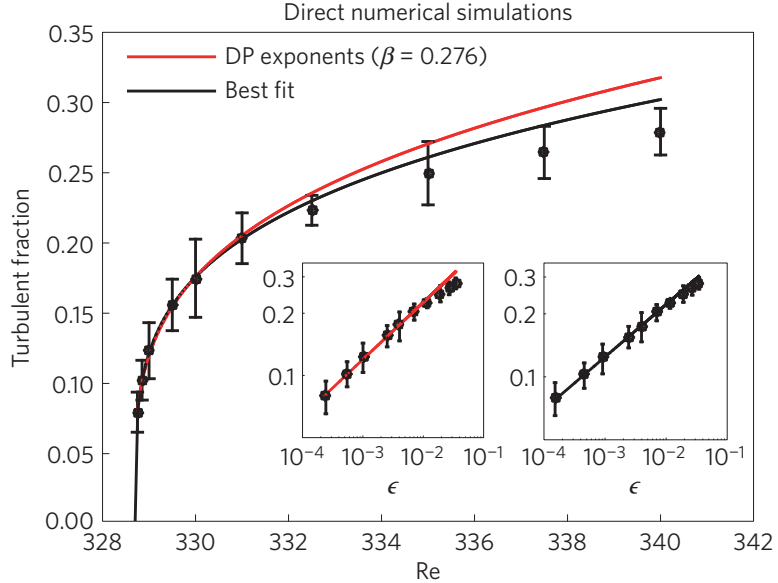

Figure 2 | Scaling of the turbulent function Equilibrium turbulent fraction as a function of Re: experiments (a) and direct numerical simulations (DNS) (b). The turbulent fraction (TF), that is, the percentage of the flow that is turbulent, is the order parameter of the transition to sustained turbulence. As the critical point $\left(\operatorname{Re}_{\mathrm{c}} \approx 328.7\right.$ in simulations and $\operatorname{Re}_{\mathrm{c}} \approx 377.5$ in experiments) is surpassed, TF increases continuously, and close to the critical point it follows a power law. In experiments the best fit results in an exponent of $\beta \sim 0.28$ (black curve in a), whereas in simulations we obtain an exponent of $\beta \sim 0.25$ (black curve in b). These values are close to the universal DP exponent (0.276) and fits with a fixed exponent of 0.276 are shown in red. In the numerical simulations, domain sizes were increased when approaching the critical point. For $\operatorname{Re}<329$, simulations were carried out in domains $1920 \mathrm{~h}$ wide (using 12,288 Fourier modes). For $329<\operatorname{Re}<334$, a domain size of $960 h$ (6,144 Fourier modes) was chosen, and for Re $>334$ a domain size of $480 h$ (3,072 Fourier modes) was chosen. 48 Fourier modes were used in the stripe direction and 32 grid points in the wall-normal direction for all runs. Errors bars represent the standard deviation.

sufficiently close to the critical point resulted in scale-invariant flow patterns, which in contrast to the aforementioned studies $^{31-34}$ is a signature of a continuous, second-order transition. In the following we report detailed experiments and extensive direct numerical simulations (DNS) for Couette flow and show that the transition to turbulence is a non-equilibrium phase transition, falling into 
a

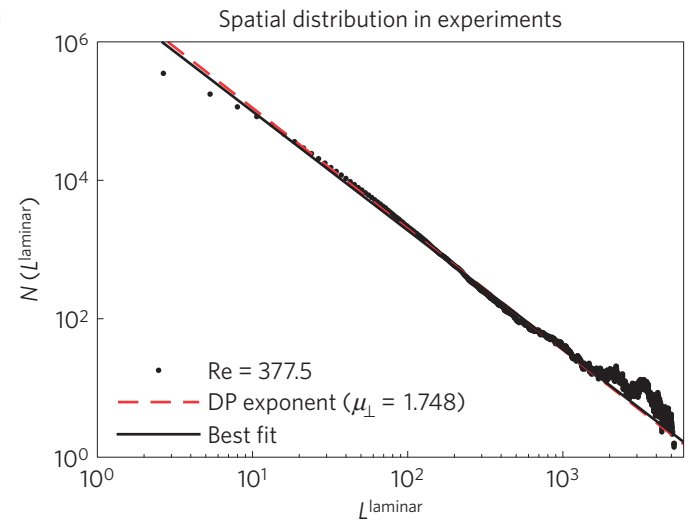

c

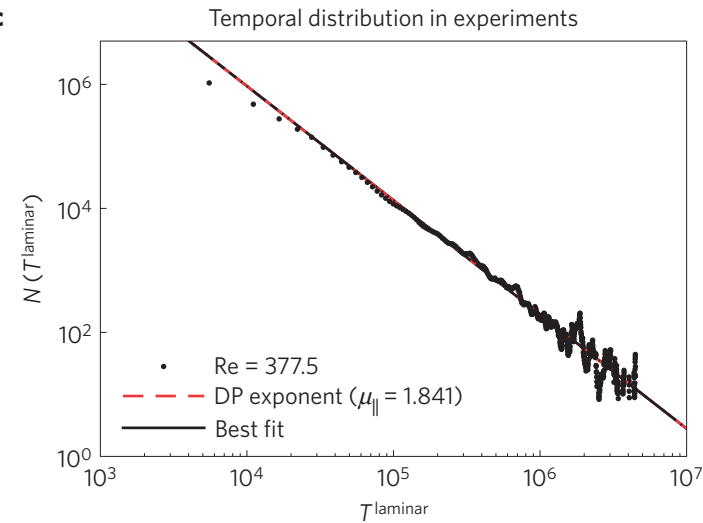

b

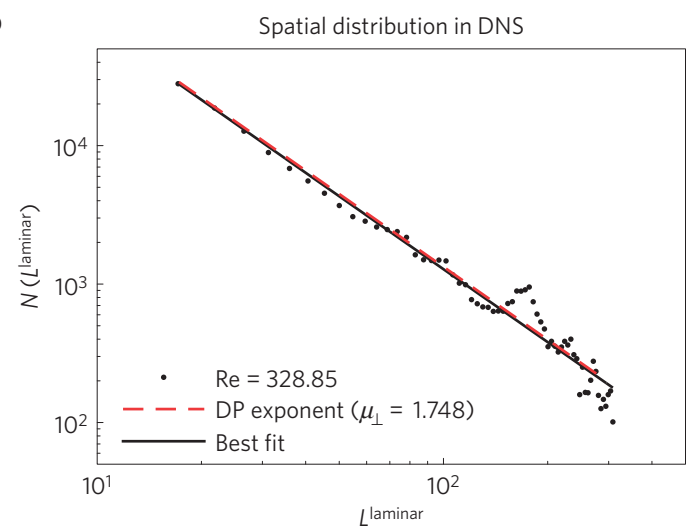

d

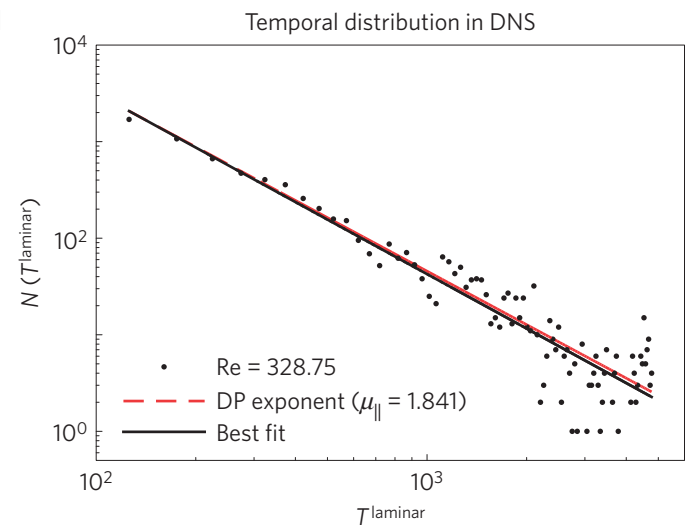

Figure 3 | Distribution of laminar gaps in space and time. The critical exponents governing the spatial and temporal correlations are determined by

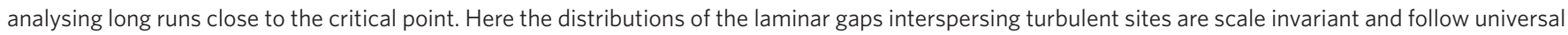
power laws (empty interval exponents). a,b, Distribution of laminar gaps ( $L^{\text {laminar}}$ ) in space from experiments (Re = 377.5) (a) and from direct numerical simulations (DNS) ( $R e=328.85)(\mathbf{b})$. The slopes correspond to the so-called spatial empty interval exponent, and the best fit to the experimental data results in a value of -1.72 , whereas in simulations we obtain -1.75 . The universal value of $D P$ is $\mu_{\perp}=1.748$, where $N(L) \sim L^{-} \mu_{\perp}$. c, d, The empty interval

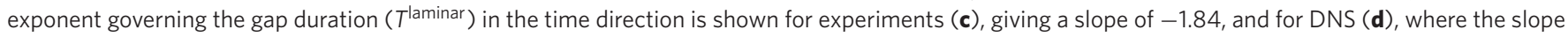
is -1.85 . The universal exponent for DP is $\mu_{\|}=1.84$, with $N(T) \sim T^{-\mu_{\|}}$and is hence in excellent agreement.

the DP universality class. Experiments are carried out in a circular Couette geometry, where the fluid is held in the gap between two concentric cylinders. An advantage of this geometry is the periodic boundary condition in the streamwise (azimuthal) direction, which in contrast to pipes and channels allows unlimited observation times. In addition to very long observation times, measurements require very large aspect ratios, as the relevant length scales are expected to diverge at the critical point. The azimuthal aspect ratio (circumference/gap) is maximized by making the radius ratio $\eta=r_{\mathrm{i}} / r_{\mathrm{o}}$ as near to unity as possible. Hence the main experimental difficulty is to keep a high relative precision of the very narrow gap (and hence Reynolds number) needed to achieve a large azimuthal aspect ratio. In our experiment, $\eta=0.998$, which is very close to the planar Couette case $(\eta=1)$ and yields an azimuthal aspect ratio of $\sim 5,500$ (see Supplementary Information for details). In the axial direction the fluid is confined by a top and a bottom end-wall (attached to the inner cylinder) and the axial aspect ratio (cylinder height $/ h$ ) is 16 . To ensure a constant Reynolds number \pm 0.25 over the long observation times, viscosity changes of the working fluid had to be minimized and hence its temperature controlled. This was achieved by circulating fluid of constant (to within $\pm 0.02 \mathrm{~K}$ ) temperature around the outside of the outer cylinder and on the inside of the inner cylinder. As working fluid, a low-viscosity (1cSt at $20^{\circ} \mathrm{C}$ ) silicone oil was chosen, which has an approximately five times smaller viscosity change with temperature in comparison to water. The current experimental set-up is a modification of a previous apparatus ${ }^{35}$ (details of the present modifications are given in the Supplementary Methods). The DNS of Couette flow was performed with the hybrid code nsCouette ${ }^{36}$ and a domain of $1,920 \times 10 \times 2$ (where the small dimension corresponds to the radial gap width) was chosen. Turbulent stripes in Couette flow are not perpendicular to the main flow (that is, azimuthal) direction. To maximize the number of stripes that can be contained in the simulated volume, we tilted the domain (by $24^{\circ}$ ) so that the long direction of the box is perpendicular to the turbulent stripes. In simulations and experiments, the elongation of the domain is essential, as it determines the number of turbulent stripes that can be accommodated. In analogy to DP, the number of stripes corresponds to the number of sites. As the distances between active sites in DP diverge when the critical point is approached, so do the laminar gaps increase between stripes in Couette flow (see Fig. 1b).

Experiments and numerical simulations followed the same procedure. First, a turbulent flow was initiated at slightly larger Re (typically $\mathrm{Re}=400$ in simulations and $\mathrm{Re}=625$ in experiments). Note that in the experiments the fluid is confined between top and bottom end-walls and, as a consequence, turbulence is observed only at higher Re than in the simulations (where periodic boundary conditions are applied). Once a turbulent flow was established, Re was reduced (quench experiments) to a value close to the critical point. The flow was then left to evolve and, after initial transients decayed, the percentage of the domain that is in turbulent motion (that is, the turbulent fraction TF) was measured and averaged over long times. TF here plays the role of the order parameter and is zero below and non-zero above the critical point (see Fig. 1b). Depending 
on whether the transition is of first or second order, TF should vary either discontinuously or continuously as the critical point is passed.

In the experiments, for each Re, the turbulent fraction was averaged over at least $10^{5}$ advective time units $\left(\mathrm{tU} \mathrm{h}^{-1}\right)$ and the regime $375<\operatorname{Re}<400$ was investigated. As shown in Fig. 2, with decreasing Re, TF decreases continuously. The given system size allowed measurements only for turbulent fractions $>\sim 15 \%$. For smaller values, fluctuations were as large as the typical number of stripes. As expected at a second-order transition, the variation of TF follows a power law, $T F \sim \varepsilon^{\beta}$ (where $\varepsilon=\left(\operatorname{Re}-\operatorname{Re}_{c}\right) / \operatorname{Re}_{c}$ and $\operatorname{Re}_{c}$ is the critical point). The exponent is very close to the universal value for directed percolation in $1+1$ dimensions (the best fit results in $\beta=0.28 \pm 0.03$, whereas the universal value for DP is 0.276 ). Likewise, in the simulations, a power law is observed and the measured exponent, $\beta=0.25 \pm 0.04$, is again close to the universal one. Although in principle an investigation of finite-size scaling, that is, the variation of the scaling relations with system size, would allow one to overcome the above restrictions, such studies pose a considerable challenge in experiments, and cannot be carried out with the present set-up.

Apart from the scaling of the order parameter, two additional critical exponents are needed; we here chose the so-called empty interval exponents, $\mu_{\perp}$ and $\mu_{\|}$, which describe the distribution of laminar/empty gaps at the critical point in space and time. The spatial exponent is determined from the slope of the distribution of laminar gap sizes at Reynolds numbers closest to the critical point (Fig. 3a,b). For the experiments $\mu_{\perp}=1.72 \pm 0.05$, whereas for the simulations $\mu_{\perp}=1.75 \pm 0.02$. These values are in very good agreement with the universal critical exponent in $\mathrm{DP}\left(\mu_{\perp}=1.748\right)$.

In analogy, the temporal empty interval exponent is determined from the distribution of laminar time intervals (Fig. 3c,d). Close to critical these should follow a power law with a slope $\mu_{\|}$. The best fit to the experimental data yielded $\mu_{\|}=1.84 \pm 0.02$, whereas in the DNS study $\mu_{\|}=1.85 \pm 0.02$ was obtained. These are in excellent agreement with the critical exponent of DP $\left(\mu_{\|}=1.84\right)$. A summary of the measured and expected exponents is given in Table 1. Hence, exponents from experiments as well as from computer simulations indicate a second-order phase transition, and the obtained values are in very good agreement with the DP universality class in $1+1 \mathrm{D}$.

In addition, a further prediction from DP regarding the time evolution of the order parameter (in our case $T F(t)$ ) could be tested for the experimental data. Starting from a fully occupied system, DP predicts that on quenching the control parameter to different values in the vicinity of the critical point, all time-series $T F(t)$ should collapse to a single curve (on a simple rescaling). As shown in Supplementary Fig. 2, the data sets indeed collapse as predicted, providing further evidence that onset of sustained turbulence in Couette flow falls into the DP framework. In principle, the collapse experiments provide an independent way of determining the critical point, and allowed us to confirm that the critical Re is within \pm 0.5 of the value determined above (Fig. 2a). However, the relatively large scatter of the data unfortunately prohibits a more accurate estimate.

Despite numerous studies, the nature of the onset of turbulence in shear flows had remained unresolved since the nineteenth century. In early studies (for example, by Kelvin, Rayleigh, Lorentz and Heisenberg ${ }^{37}$ ) attempts were limited to linear stability analysis, which cannot explain the sub-critical transition scenario common to these flows. Although concepts from statistical physics have been discussed in this context for 30 years, earlier studies underestimated the spatial and temporal scales relevant for the transition process. We speculate that the onset of sustained turbulence in pipe and other shear flows will equally fall into the DP universality class. In situations where turbulence can spread in two directions (such studies would require Couette or channel experiments with lengths and widths comparable to the domain length used in the present
Table 1/ Comparison between the critical exponents measured in Couette experiments, Couette simulations and the exponents of the DP universality class.

\begin{tabular}{llll} 
Critical exponents & $\boldsymbol{\mu}_{\perp}$ & $\boldsymbol{\mu}_{\|}$ & $\boldsymbol{\beta}$ \\
\hline Couette simulations & $1.75 \pm 0.02$ & $1.85 \pm 0.02$ & $0.25 \pm 0.04$ \\
Couette experiments & $1.72 \pm 0.05$ & $1.84 \pm 0.02$ & $0.28 \pm 0.03$ \\
DP & 1.748 & 1.84 & 0.276 \\
\hline
\end{tabular}

(Note that the empty interval exponents are directly related to the more commonly used spatial and temporal correlation exponents $v_{\perp}, v_{\|}$by $\mu_{\perp / \|}=2-\beta / v_{\perp / \|}$. ) Error estimates correspond to $95 \%$ confidence intervals.

study) the exponents are likely to change to DP in $2+1$ dimensions (see also ref. 38), although discontinuous transitions cannot be ruled out at this stage. Demonstrating that models from non-equilibrium statistical mechanics can explain phenomena as complex as the onset of turbulence, and that universal critical exponents can be explicitly measured, should inspire further research exploring universality far from equilibrium.

Received 15 October 2015; accepted 25 January 2016; published online 15 February 2016

\section{References}

1. Avila, K. et al. The onset of turbulence in pipe flow. Science 333, 192-196 (2011).

2. Shi, L., Avila, M. \& Hof, B. Scale invariance at the onset of turbulence in Couette flow. Phys. Rev. Lett. 110, 204502 (2013)

3. Wang, J., Li, Q. \& E, W. Study of the instability of the Poiseuille flow using a thermodynamic formalism. Proc. Natl Acad. Sci. USA 112, 9518-9523 (2015).

4. Reynolds, O. An experimental investigation of the circumstances which determine whether the motion of water shall be direct or sinuous, and of the law of resistance in parallel channels. Proc. R. Soc. Lond. 35, 84-99 (1883).

5. Coles, D. Transition in circular Couette flow. J. Fluid Mech. 21, 385-425 (1965).

6. Barkley, D. et al. The rise of fully turbulent flow. Nature 526, 550-553 (2015).

7. Orr, W. M. F. The stability or instability of the steady motions of a perfect liquid and a viscous liquid. Proc. R. Irish Acad. A 27, 69-138 (1907).

8. Sommerfeld, A. Proceedings of the 4th International Mathematical Congress, Rome 1908 116-124 (1909).

9. Lorentz, A. Über die Entstehung turbulenter Flüssigkeitsbewegungen und über den Einfluss dieser Bewegungen bei der Strömung durch Röhren. Abhandlungen über Theor. Phys. Leipzig 1, 43-71 (1907).

10. Heisenberg, W. Über Stabilität und Turbulenz von Flüssigkeitsströmen. Ann. Phys. 74, 577-627 (1924).

11. Trefethen, L. N., Trefethen, A. E., Reddy, S. C. \& Driscoll, T. A. Hydrodynamic stability without eigenvalues. Science. 261, 578-584 (1993).

12. Henningson, D. \& Reddy, S. On the role of linear mechanisims in transition to turbulence. Phys. Fluids 6, 1396-1398 (1994).

13. Nagata, M. Three-dimensional finite-amplitude solutions in plane Couette flow: bifurcation from infinity. J. Fluid Mech. 217, 519-527 (1990).

14. Faisst, H. \& Eckhardt, B. Traveling waves in pipe flow. Phys. Rev. Lett. 91, 224502 (2003)

15. Wedin, H. \& Kerswell, R. R. Exact coherent structures in pipe flow: travelling wave solutions. J. Fluid Mech. 508, 333-371 (2004).

16. Hof, B. et al. Experimental observation of nonlinear traveling waves in turbulent pipe flow. Science 305, 1594-1598 (2004).

17. Gibson, J. F., Halcrow, J. \& Cvitanović, P. Equilibrium and traveling-wave solutions of plane Couette flow. J. Fluid Mech. 638, 1-24 (2009).

18. Avila, M., Mellibovsky, F., Roland, N. \& Hof, B. Streamwise-localized solutions at the onset of turbulence in pipe flow. Phys. Rev. Lett. 110, 224502 (2013).

19. Hinrichsen, $H$. Non-equilibrium critical phenomena and phase transitions into absorbing states. Adv. Phys. 49, 815-958 (2000).

20. Takeuchi, K. A. \& Sano, M. Universal fluctuations of growing interfaces: evidence in turbulent liquid crystals. Phys. Rev. Lett. 104, 230601 (2010).

21. Almeida, R. A. L. et al. Universal fluctuations in the growth of semiconductor thin films. Phys. Rev. B 89, 045309 (2014).

22. Pomeau, Y. Front motion, metastability and subcritical bifurcations in hydrodynamics. Physica D 23, 3-11 (1986).

23. Janssen, $H$. On the nonequilibrium phase transition in reaction-diffusion system with an absorbing stationary state. Z. Phys. B 42, 151-154 (1981). 
24. Grassberger, P. On phase transitions in Schlögl's second model. Z. Phys. B 47, 365-374 (1982)

25. Takeuchi, K. A., Kuroda, M., Chate, H. \& Sano, M. Directed percolation criticality in turbulent liquid crystals $\rho$. Phys. Rev. Lett. 99, 234503 (2007).

26. Chate, H. \& Manneville, P. Spatio-temproal intermittency in coupled map lattices. Physica D 32, 409-422 (1988)

27. Rolf, J., Bohr, T. \& Jensen, M. H. Directed percolation universality in asynchronous evolution of spatiotemporal intermittency. Phys. Rev. E 57, R2503-R2506 (1998)

28. Sipos, M. \& Goldenfeld, N. Directed percolation describes lifetime and growth of turbulent puffs and slugs. Phys. Rev. E 84, 035304 (2011).

29. Barkley, D. Simplifying the complexity of pipe flow. Phys. Rev. E 84, 016309 (2011).

30. Shih, H.-Y., Hsieh, T.-L. \& Goldenfeld, N. Ecological collapse and the emergence of traveling waves at the onset of shear turbulence. http://dx.doi.org/10.1038/nphys3548 (2015).

31. Bottin, S., Daviaud, F., Manneville, P. \& Dauchot, O. Discontinuous transition to spatiotemporal intermittency in plane Couette flow. Eur. Lett. 43, 171-176 (1998)

32. Bottin, S. \& Chate, H. Statistical analysis of the transition to turbulence in plane Couette flow. Eur. Phys. J. B 6, 143-155 (1998).

33. Duguet, Y., Schlatter, P. \& Henningson, D. S. Formation of turbulent patterns near the onset of transition in plane Couette flow. J. Fluid Mech. 650, 119-129 (2010).

34. Manneville, P. Spatiotemporal perspective on the decay of turbulence in wall-bounded flows. Phys. Rev. E 79, 025301 (2009).

35. Avila, K. \& Hof, B. High-precision Taylor-Couette experiment to study subcritical transitions and the role of boundary conditions and size effects. Rev. Sci. Instrum. 84, 065106 (2013).

36. Shi, L., Rampp, M., Hof, B. \& Avila, M. A hybrid MPI-OpenMP parallel implementation for pseudospectral simulations with application to Taylor-Couette flow. Comput. Fluids 106, 1-11 (2015).
37. Eckert, M. The troublesome birth of hydrodynamic stability theory: sommerfeld and the turbulence problem. Eur. Phys. J. H 35, 29-51 (2010).

38. Sano, M. \& Tamai, K. A universal transition to turbulence in channel flow. Preprint at http://arXiv.org/abs/1510.07868 (2015).

\section{Acknowledgements}

We thank P. Maier for providing valuable ideas and supporting us in the technical aspects. Discussions with D. Barkley, Y. Duguet, B. Eckhart, N. Goldenfeld, P. Manneville and $\mathrm{K}$. Takeuchi are gratefully acknowledged. We acknowledge the Deutsche Forschungsgemeinschaft (Project No. FOR 1182), and the European Research Counci under the European Union's Seventh Framework Programme (FP/2007-2013)/ERC Grant Agreement 306589 for financial support. L.S. and B.H. acknowledge research funding by Deutsche Forschungsgemeinschaft (DFG) under Grant No. SFB 963/1 (project A8). Numerical simulations were performed thanks to the CPU time allocations of JUROPA in Juelich Supercomputing Center (project HGU17) and of the Max Planck Computing and Data Facility (Garching, Germany). Excellent technical support from M. Rampp on the hybrid code nsCouette is appreciated.

\section{Author contributions}

G.L., K.A. and B.H. designed the experiment. K.A. and S.V.J. performed preliminar experiments. G.L. performed final experiments. B.H. supervised the experimental work. L.S. and M.A. designed and performed the computer simulations of the Navier-Stokes equations. M.A. and B.H. supervised the computer simulations. G.L., L.S., K.A., S.V.J., M.A. and B.H. wrote the paper.

\section{Additional information}

Supplementary information is available in the online version of the paper. Reprints and permissions information is available online at www.nature.com/reprints.

Correspondence and requests for materials should be addressed to B.H.

\section{Competing financial interests}

The authors declare no competing financial interests. 